\title{
Incidencia de herpes zóster en pacientes diabéticos
}

\section{Herpes zoster incidence in diabetic patients}

\author{
P. Aldaz ${ }^{1,2,3}$, J.A. Díaz ${ }^{4}$, J.R. Loayssa ${ }^{5}$, M.J. Dronda ${ }^{1}$, M. Osacáriz ${ }^{1,3}$, J. Castilla ${ }^{6,7}$
}

\section{RESUMEN}

Objetivo. Evaluar el riesgo de herpes zóster en personas con diabetes mellitus en comparación con el resto de la población.

Métodos. A partir de la información de historia clínica informatizada de atención primaria de Navarra se ha analizado la incidencia de herpes zóster en mayores de 30 años en 2006 en función del diagnóstico de diabetes, y ajustando por edad y sexo.

Resultados. En 26.793 diabéticos se diagnosticaron 409 casos de herpes zóster (15 por mil). El 19,3\% de los casos se produjeron en diabéticos. La diabetes presentó un riesgo relativo ajustado de 2,1 (IC 95\% 1,9-2,4), y en el grupo de 30 a 44 años ascendió a 3,7 (IC 95\% 2,0-6,8). El 7,2\% de todos los casos de herpes zóster en la población fueron atribuibles a la diabetes.

Conclusiones. La diabetes aumenta el riesgo y tiene un peso importante en la incidencia de herpes zóster.

Palabras clave. Herpes zóster. Diabetes mellitus. Virus varicela-zóster. Incidencia. España.

\begin{abstract}
Background. To assess the risk of herpes zoster in people with diabetes mellitus in comparison with the general population.

Methods. Using computerized clinical reports form primary health care in Navarre, Spain, we analyzed the incidence of herpes zoster in people aged over 30 years in 2006 by diagnosis of diabetes, and adjusting for age and sex.

Results. Among 26,793 diabetes patients 409 cases of herpes zoster were diagnosed (15 per 1000). Nineteen point three percent of cases occurred in diabetes patients. Diabetes presented an adjusted relative risk of 2.1 (95\% CI 1.92.4), and increased to 3.7 (95\% CI 2.0-6.8) in the $30-44$ year age group. Of the total number of cases of herpes zoster in the population, $7.2 \%$ were attributable to diabetes.
\end{abstract}

Conclusion. Diabetes increases the risk of, and has a relevant role in, the incidence of herpes zoster.

Key words. Herpes zoster. Diabetes mellitus. Varicellazoster virus. Incidence. Spain.
1. Centro de Salud de San Juan. Pamplona.

2. Grupo de Prevención de Enfermedades Infecciosas, PAPPS-SEMFYC.

3. Grupo de Enfermedades Infecciosas, SNAMFAP.

4. Centro de Salud II Ensanche. Pamplona.

5. Centro de Salud de Huarte. Navarra

6. Instituto de Salud Pública de Navarra. Pamplona.

7. CIBER de Epidemiología y Salud Pública.

\section{Correspondencia:}

Pablo Aldaz Herce

Centro de Salud San Juan

Plaza Obispo Irurita, $\mathbf{s} / \mathbf{n}$

31011 Pamplona

E-mail: paldazhe@cfnavarra.es

Recepción: 25 de julio de 2012

Aceptación provisional: 3 de septiembre de 2012 Aceptación definitiva: 12 de septiembre de 2012 


\section{INTRODUCCIÓN}

El herpes zóster es causado por el virus varicela-zóster, que tras la varicela puede permanecer acantonado en los ganglios espinales o del trigémino y reactivarse en situaciones de disminución de la inmunidad ${ }^{1}$ La incidencia aumenta con la $\operatorname{edad}^{2-3}$, y en enfermedades y situaciones que afectan a la inmunidad celular.

Desde finales de los noventa se ha ido extendiendo la vacunación de la varicela en niños, y algunas comunidades autónomas la han introducido en el calendario de vacunaciones sistemáticas, lo cual está modificando la circulación del virus en la población y puede afectar a la incidencia de herpes zóster ${ }^{4}$

La diabetes mellitus es una enfermedad con alta prevalencia en la población adulta, y suele acompañarse de un grado variable de depresión del sistema inmune. Cabe esperar que los pacientes diabéticos tengan un riesgo aumentado de herpes zóster, si bien este aspecto ha sido poco estudiado ${ }^{5}$.

Se dispone de una vacuna eficaz para prevenir el herpes zóster ${ }^{6}$, por lo que tiene interés identificar los factores de riesgo que puedan orientar sobre prioridades para la vacunación.

El objetivo del presente estudio es conocer el riesgo de herpes zóster en personas con diagnóstico de diabetes y compararlo con el del resto de la población.

\section{MÉTODOS}

Realizamos un estudio observacional utilizando la información registrada en la historia clínica informatizada de Navarra, la cual está implantada en todos los centros de atención primaria desde el año 2005. Los médicos de atención primaria han de asignar un código diagnóstico de la clasificación internacional de atención primaria, segunda versión, a todos los procesos nuevos que atienden y esta información se almacena en un sistema informático centralizado.

El presente estudio se centró en personas de 30 años o más, por la escasa incidencia de herpes zóster en menores de esta edad, y abarcó el año 2006, en el que todavía no se había extendido la vacunación frente a la varicela. La población se estratificó según sexo, grupos de edad y diagnóstico de diabetes. Se consideraron diagnosticados de diabetes mellitus los pacientes que tenían asignados los códigos T89 (diabetes tipo 1) o T90 (diabetes tipo 2). De la misma fuente de información se tomaron los diagnósticos de herpes zóster realizados durante 2006, considerando aquellos que tenían asignado el código S72 de la clasificación internacional. Para este análisis solo se utilizaron datos anónimos y agregados.

Se calcularon tasas de incidencia de herpes zóster, crudas y estratificadas por edad y sexo, para personas con y sin diagnóstico de diabetes. Se comparó la incidencia en ambos grupos calculando riesgos relativos con sus intervalos de confianza (IC) al 95\%. Se utilizó regresión de Poisson para comparar el riesgo de herpes zóster entre personas con y sin diagnóstico de diabetes, ajustando por edad y sexo. En los modelos se evaluaron los términos de interacción del sexo y la edad con el diagnóstico de diabetes.

Por último, se calculó la proporción de casos de herpes zóster en la población que eran atribuibles a la diabetes. Los análisis fueron realizados con Stata versión 11.

\section{RESULTADOS}

En 2006 se registraron en Navarra 2.121 casos de herpes zóster en personas de 30 años o más $(5,4$ por mil habitantes y año; IC $95 \% 5,2-5,7)$, siendo la tasa menor en hombres $(4,6$ por mil) que en mujeres $(6,2$ por mil; $\mathrm{p}<0,001)$. En la población sin diagnóstico previo de diabetes se registraron 1.712 casos de herpes zóster (4,7 por mil; IC 95\% $4,4-4,9)$, con tasas de 3,9 por mil en hombres y 5,4 por mil en mujeres.

El 6,8\% ( $n=26.793)$ de la población tenía diagnóstico de diabetes, y entre ellos se registraron el 19,3\% de los casos de herpes zóster ( $\mathrm{n}=409)$. La tasa de herpes zóster en esta población fue de 15,3 por mil (IC $95 \%$ $13,8-16,8)$, siendo de 18,7 por mil en mujeres y 12,4 por mil en hombres. Tanto en población sin diagnóstico de diabetes como en pacientes diabéticos la incidencia de herpes zóster aumentó con la edad (Tabla 1). 
Tabla 1. Incidencia de herpes zóster en población con y sin diagnóstico de diabetes mellitus. Navarra, 2006

\begin{tabular}{|c|c|c|c|c|c|c|c|c|c|}
\hline \multirow{2}{*}{ Sexo y edad } & \multicolumn{3}{|c|}{ Sin diagnóstico de diabetes } & \multicolumn{3}{|c|}{ Con diagnóstico de diabetes } & \multicolumn{3}{|c|}{ Comparación } \\
\hline & $\mathbf{N}$ & Casos & $\begin{array}{c}\text { Tasa por } \\
10^{3}\end{array}$ & $\mathbf{N}$ & Casos & $\begin{array}{c}\text { Tasa } \\
\text { por } 10^{3}\end{array}$ & $\begin{array}{c}\text { Riesgo } \\
\text { relativo }\end{array}$ & IC $95 \%$ & $\mathbf{P}$ \\
\hline \multicolumn{10}{|l|}{ Hombres } \\
\hline $30-44$ & 76.475 & 143 & 1,9 & 981 & 6 & 6,1 & 3,3 & $1,5-7,4$ & 0,012 \\
\hline $45-59$ & 53.773 & 186 & 3,5 & 3.650 & 26 & 7,1 & 2,1 & $1,4-3,1$ & 0,002 \\
\hline $60-74$ & 32.222 & 221 & 6,9 & 6.340 & 85 & 13,4 & 2,0 & $1,5-2,5$ & $<0,001$ \\
\hline$\geq 75$ & 16.575 & 151 & 9,1 & 3.554 & 63 & 17,7 & 2,0 & $1,5-2,6$ & $<0,001$ \\
\hline Total & 179.045 & 701 & 3,9 & 14.525 & 180 & 12,4 & 3,2 & $2,7-3,7$ & $<0,001$ \\
\hline \multicolumn{10}{|l|}{ Mujeres } \\
\hline $30-44$ & 70.094 & 135 & 1,9 & 588 & 5 & 8,5 & 4,4 & $1,8-10,8$ & 0,007 \\
\hline $45-59$ & 53.798 & 299 & 5,6 & 1.795 & 23 & 12,8 & 2,3 & $1,5-3,5$ & $<0,001$ \\
\hline $60-74$ & 36.868 & 334 & 9,1 & 4.657 & 77 & 16,5 & 1,8 & $1,4-2,3$ & $<0,001$ \\
\hline$\geq 75$ & 27.387 & 243 & 8,9 & 5.228 & 124 & 23,7 & 2,7 & $2,2-3,3$ & $<0,001$ \\
\hline Total & 188.147 & 1011 & 5,4 & 12.268 & 229 & 18,7 & 3,5 & $3,0-4,0$ & $<0,001$ \\
\hline \multicolumn{10}{|l|}{ Ambos sexos } \\
\hline $30-44$ & 146.569 & 278 & 1,9 & 1.569 & 11 & 7,0 & 3,7 & $2,0-6,8$ & $<0,001$ \\
\hline $45-59$ & 107.571 & 485 & 4,5 & 5.445 & 49 & 9,0 & 2,0 & $1,5-2,7$ & $<0,001$ \\
\hline $60-74$ & 69.090 & 555 & 8,0 & 10.997 & 162 & 14,7 & 1,8 & $1,5-2,2$ & $<0,001$ \\
\hline$\geq 75$ & 43.962 & 394 & 9,0 & 8.782 & 187 & 21,3 & 2,4 & $2,0-2,8$ & $<0,001$ \\
\hline Total & 367.192 & 1712 & 4,7 & 26.793 & 409 & 15,3 & 3,3 & $2,9-3,7$ & $<0,001$ \\
\hline
\end{tabular}

En una comparación cruda, la incidencia de herpes zóster fue 3,3 veces mayor (IC 95\% 2,9-3,7) en personas con diagnóstico de diabetes. En todos los grupos de edad y en ambos sexos el diagnóstico de diabetes multiplicó al menos por 1,8 la incidencia de herpes zóster, si bien, este aumento fue mayor en los de 30 a 44 años, donde se multiplicó por 3,7. En personas de 30 a 44 años la diabetes elevó el riesgo de herpes zóster ( 7,0 por mil) a niveles que sólo se alcanzan a partir de los 60 años entre los no diabéticos.
En el análisis multivariante, el sexo femenino, el aumento de edad y el diagnóstico de diabetes mostraron efectos independientes elevando el riesgo de herpes zóster (Tabla 2). La diabetes presentó un riesgo relativo de 2,1 (IC 95\% 1,9-2,4). Este riesgo relativo asociado a la diabetes fue mayor en personas de 30 a 44 años (3,7; IC 95\% 2,0$6,8)$ que en los mayores de esta edad $(2,1 ;$ IC $95 \%$ 1,9-2,4), diferencia respaldada por una interacción próxima a la significación estadística $(\mathrm{p}=0,061)$. 
Tabla 2. Evaluación del efecto de la edad, del sexo y del diagnóstico de diabetes mellitus sobre el riesgo de herpes zóster. Navarra, 2006

\begin{tabular}{|c|c|c|c|c|}
\hline & Riesgo relativo crudo & IC $95 \%$ & Riesgo relativo ajustado & IC $95 \%$ \\
\hline \multicolumn{5}{|c|}{ Población $\geq 30$ años } \\
\hline \multicolumn{5}{|l|}{ Edad, años } \\
\hline $30-44$ & 1 & & 1 & \\
\hline $45-59$ & 2,4 & $2,1-2,8$ & 2,3 & $2,0-2,7$ \\
\hline $60-74$ & 4,6 & $4,0-5,3$ & 4,0 & $3,5-4,6$ \\
\hline$\geq 75$ & 5,7 & $4,9-6,5$ & 4,6 & $4,0-5,4$ \\
\hline \multicolumn{5}{|l|}{ Sexo } \\
\hline Hombre & 1 & & 1 & \\
\hline Mujer & 1,4 & $1,3-1,5$ & 1,3 & $1,2-1,4$ \\
\hline \multicolumn{5}{|c|}{ Diagnóstico de diabetes } \\
\hline No & 1 & & 1 & \\
\hline Sí & 3,3 & $2,9-3,7$ & 2,1 & $1,9-2,4$ \\
\hline \multicolumn{5}{|c|}{ Población de 30-44 años } \\
\hline \multicolumn{5}{|l|}{ Sexo } \\
\hline Hombre & 1 & & 1 & \\
\hline Mujer & 1,0 & $0,8-1,3$ & 1,0 & $0,8-1,3$ \\
\hline \multicolumn{5}{|c|}{ Diagnóstico de diabetes } \\
\hline No & 1 & & 1 & \\
\hline Sí & 3,7 & $2,0-6,8$ & 3,7 & $2,0-6,8$ \\
\hline \multicolumn{5}{|c|}{ Población $\geq 45$ años } \\
\hline \multicolumn{5}{|l|}{ Edad, años } \\
\hline $45-59$ & 1 & & 1 & \\
\hline $60-74$ & 1,9 & $1,7-2,1$ & 1,7 & $1,5-1,9$ \\
\hline$\geq 75$ & 2,3 & $2,1-2,6$ & 2,0 & $1,8-2,3$ \\
\hline \multicolumn{5}{|l|}{ Sexo } \\
\hline Hombre & 1 & & 1 & \\
\hline Mujer & 1,4 & $1,2-1,5$ & 1,3 & $1,2-1,5$ \\
\hline \multicolumn{5}{|c|}{ Diagnóstico de diabetes } \\
\hline No & 1 & & 1 & \\
\hline Sí & 2,4 & $2,2-2,7$ & 2,1 & $1,9-2,4$ \\
\hline
\end{tabular}

Dada la prevalencia de diabetes $(6,8 \%)$ y el riesgo relativo ajustado se estima que el $7,2 \%$ de todos los casos de herpes zóster en la población fueron atribuibles a la diabetes.

\section{DISCUSIÓN}

Los resultados muestran mayor incidencia de herpes zóster en población dia- bética en comparación con la población sin diabetes de igual edad y sexo.

Coincidiendo con otros trabajos, encontramos que el riesgo de herpes zóster aumenta progresivamente con la edad ${ }^{7}$, siendo este el principal predictor de la enfermedad, lo cual puede explicarse por la immunosenescencia ${ }^{8}$. Detectamos mayor incidencia en las mujeres que en los hombres a partir de los 45 años de edad, sin que 
se haya podido explicar hasta el momento la causa de estas diferencias ${ }^{9}$

Independientemente de la edad y del sexo, la diabetes es un predictor importante del riesgo de desarrollar herpes zóster. Entre los diabéticos, a partir de 30 años de edad se alcanzaron tasas de herpes zóster que solo se observan en población no diabética a partir de los 60 años. La diabetes puede afectar a la inmunidad celular por diferentes mecanismos y puede facilitar la reactivación del virus varicela-zóster que permanece acantonado.

El riesgo elevado de herpes zóster en diabéticos coincide con lo descrito en otros estudios ${ }^{10-11}$ En la población estudiada, uno de cada cinco casos de herpes zóster se produjeron en personas con diagnóstico de diabetes, lo que demuestra su importante repercusión clínica y el gasto sanitario asociado ${ }^{12}$

El exceso de riesgo de herpes zóster en pacientes diabéticos fue más pronunciado en jóvenes, en quienes, salvo que exista alguna patología, la inmunidad está preservada. A partir de los 45 años el exceso de riesgo asociado a la diabetes se redujo, lo cual puede deberse al aumento de otras enfermedades concurrentes que contribuirían a reducir las diferencias entre la respuesta inmune de diabéticos y no diabéticos.

El presente estudio está sujeto a algunas limitaciones. La información procede de la historia clínica informatizada, y aunque en 2006 estaba implantada en toda la red de atención primaria de Navarra, no podemos descartar cierta infraestimación, tanto de diabetes como de herpes zóster, ya que los casos que tuvieran asignados códigos distintos de los que hemos analizado no se habrán incluido. Consideramos únicamente los casos de diabetes diagnosticados, a pesar de que proporciones considerables de los casos permanecen sin diagnosticar. El grupo de comparación fue la población sin diagnóstico de diabetes, la cual incluye personas con otras patologías concurrentes que pueden ser factores de riesgo para el herpes zóster. Todas estas limitaciones tenderían infraestimar el riesgo relativo asociado a la diabetes.
En conclusión, la población diabética presenta un riesgo elevado de herpes zóster y contabiliza una proporción considerable de todos los casos. La aplicación de cualquier medida preventiva o terapéutica frente al herpes zóster, incluida la vacuna, debería considerar a la población diabética entre sus prioridades.

\section{Agradecimientos}

A la Dirección de Atención Primaria de Navarra por facilitarnos los datos para la elaboración de este trabajo y a todos los compañeros de Atención Primaria por participar en la cumplimentación de las historias clínicas informatizadas.

\section{BIBLIOGRAFÍA}

1. Mueller NH, Gilden DH, Cohrs RJ, Mahalingam R, NAGEL MA. Varicella zoster virus infection: clinical features, molecular pathogenesis of disease, and latency. Neurol Clin 2008; 26: 675-697.

2. Cebrian-Cuenca AM, Díez-Domingo J, San Martín M, Puig-Barberá J, Navarro-Pérez J. Epidemiology of herpes zoster infection among patients treated in primary care centres in Valencian Community (Spain). BMC Fam Pract 2010; 6: 11-33.

3. García Cenoz M, Castilla J, Montes Y, Morán J, SAlABERri A, Elía F et al. Varicella and herpes zoster incidence prior to the introduction of systematic child vaccination in Navarre, 2005-2006. An Sist Sanit Navar 2008; 31: 7180.

4. Reynolds MA, Chaves SS, Harpaz R, Lopez AS, SEWARD JF. The impact of the varicella vaccination program on herpes zoster epidemiology in the United States: a review. J Infect Dis 2008; 197: S224-227.

5. Nassaji-Zavareh M, Taheri R, GHorbani R, Aminian M. Undiagnosed diabetes mellitus in patients with Herpes Zoster. Indian J Dermatol 2008; 53: 119-121.

6. Oxman MN, Levin MJ, Johnson GR, Schmader KE, Straus SE, Gelb LD et al. A vaccine to prevent herpes zoster and postherpetic neuralgia in older adults. N Engl J Med 2005; 352:22712284.

7. Gil A, San-Martín M, Carrasco P, González A. Epidemiology of severe varicella-zoster virus infection in Spain. Vaccine 2004; 22: 39473951. 
8. Oxman MN. Herpes zoster pathogenesis and cell-mediated immunity and immunosenescence. J Am Osteopath Assoc 2009; 109: S1317.

9. Opstelten W, Van Essen Ga, Schellevis F, VerheiJ TJ, Moons KG. Gender as an independent risk factor for herpes zoster: a population-based prospective study. Ann Epidemiol 2006; 16: 692-695.

10. Окамoto S, Hata A, Sadaoka K, Yamanishi K, MoRI Y. Comparison of varicella-zoster virusspecific immunity of patients with diabetes mellitus and healthy individuals. J Infect Dis 2009; 200: 1606-1610.

11. Heymann AD, Chodick G, Karpati T, Kamer L, Kremer E, Green MS et al. Diabetes as a risk factor for herpes zoster infection: results of a population-based study in Israel. Infection 2008; 36: 226-230.

12. JiH JS, CHeN YJ, LIN MW, CHEN YC, CHEN TJ, HuANG YL et al. Epidemiological features and costs of herpes zoster in Taiwan: a national study 2000 to 2006. Acta Derm Venereol 2009; 89: 612-616. 